\title{
Identification of Simulated Moving Bed Dynamic System by Neural Network
}

\author{
I-Chun Chen, ${ }^{1}$ Huang-Chu Huang, ${ }^{2}$ Chi-Yen Shen, ${ }^{1}$ and Rey-Chue Hwang ${ }^{1 *}$ \\ ${ }^{1}$ Department of Electrical Engineering, I-Shou University, \\ No. 1, Sec. 1, Syuecheng Rd., Dashu District, Kaohsiung City 84001, Taiwan \\ ${ }^{2}$ Department of Telecommunication Engineering, National Kaohsiung University of Science and Technology, \\ No. 142, Haijhuan Rd., Nanzih District, Kaohsiung City 81157, Taiwan
}

(Received December 13, 2019; accepted September 8, 2020)

Keywords: system identification, SMB, neural network

This paper presents a study about the possibility of system identification for a simulated moving bed (SMB), which is an important step for developing a smart SMB automatic control mechanism with a precise control capability. An SMB is a very complex and nonlinear system that is constructed from multiple columns in series and complex valve arrangements. All feed mixtures and solvents and the desorbent flow are controlled by the columns and valve devices at a fixed switching time. Thus, if the operational behavior of an SMB system can be identified in advance, then the precise control of the system can be achieved easily. In this study, the neural network (NN) technique was used to identify an SMB system. From the experimental results shown, an NN was found to be a very effective tool for SMB system identification.

\section{Introduction}

A simulated moving bed (SMB) is well known as an effective tool for highly precise chromatographic separation processes. It is capable of separating one chemical compound or a class of chemical compounds from one or more other compounds. SMBs have been widely used in the chemical and biomedical industries to obtain significant quantities of purified materials at a lower manufacturing cost. Owing to the reduced amounts of solvent and energy needed, the SMB is recognized as the cleanest technique in the application of chromatography. Basically, an SMB system is composed of several identical columns, which are connected in series to form a continuous loop. Figure 1 shows an example of the operation process of an SMB with eight columns. The entire operation process includes the feeding of a mixture, the output of purified components, and the input of a solvent or eluent. ${ }^{(1-8)}$

To increase the production capacity of SMBs, the precise control of multicolumn SMBs has become a hot topic and a problem that current researchers want to solve. However, the physical behavior of an SMB system is complex and nonlinear, making it very difficult to build a mathematical model for controlling an SMB system. Most of the studies on SMB systems used an approximate model as a reference to implement the actual system control. ${ }^{(9,10)}$ Because such

*Corresponding author: e-mail: rchwang@isu.edu.tw https://doi.org/10.18494/SAM.2021.2736 


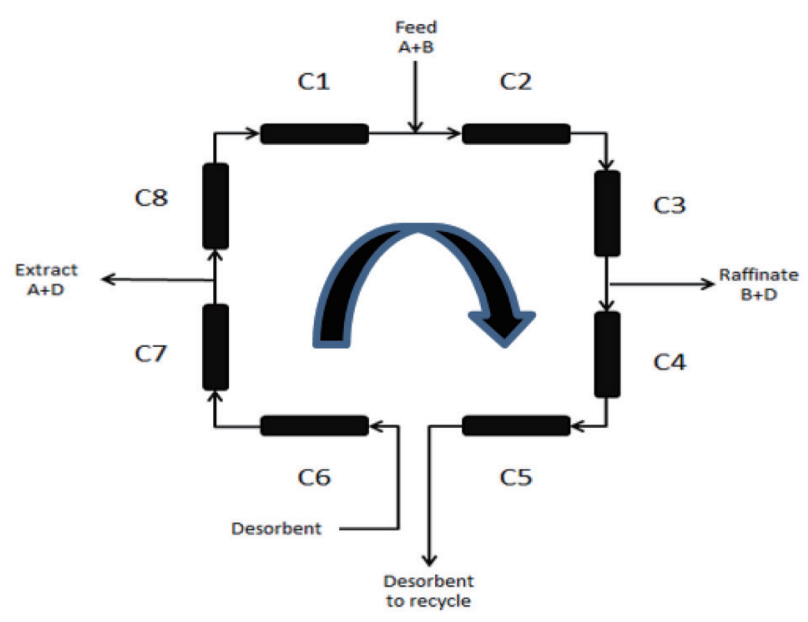

Fig. 1. (Color online) Example of SMB operation process.

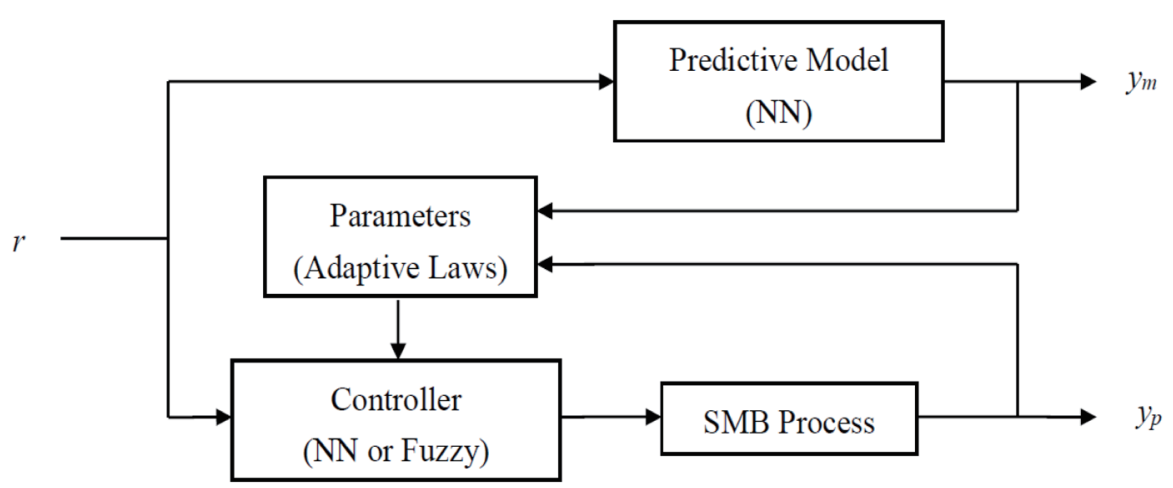

Fig. 2. Entire SMB control process.

a control mechanism design is only based on an approximation model, it cannot truly reflect the real state of an SMB. Thus, it is not yet possible to achieve the effective and precise control of an SMB system.

Owing to the learning capability of neural networks (NNs), a well-trained NN can be used to capture the complex relationship between SMB control variables and the concentration of individually separated substances, enabling the accurate prediction of the concentration of individual substances in a separated mixture. In other words, such a well-trained NN can replace a real SMB system when developing a smart SMB automatic control mechanism with precise controllability. The entire SMB control process is shown in Fig. 2.

To capture the actual operation conditions of an SMB system, three flow sensors and two concentration sensors could be used in experiments to collect the data of the SMB separation process. These data reflect the actual behavior of the SMB during separation and can be used to identify SMB systems employing an NN model. 


\section{SMB Model}

The operation of an SMB is mainly derived from the concept of the true moving bed (TMB). Their mathematical models can be expressed as follows. ${ }^{(11,12)}$ For a TMB, the mass balance of the bulk phase is

$$
\begin{gathered}
\frac{\partial C_{i j}}{\partial t}=D_{i} \frac{\partial^{2} C_{i j}}{\partial x^{2}}-v_{j}^{*} \frac{\partial C_{i j}}{\partial x}-\frac{1-\varepsilon}{\varepsilon} k_{i}\left(q_{i j}^{*}-q_{i j}\right), \\
\frac{\partial q_{i j}}{\partial t}=\frac{\partial}{\partial x} u_{s} q_{i j}+k_{i}\left(q_{i j}^{*}-q_{i j}\right) .
\end{gathered}
$$

Here, $i$ is the $i$-th component of mixing, $j$ is the $j$-th segment, $x$ is the distance in the moving direction, $k_{i}$ is a comprehensive mass transfer constant, $v_{j}^{*}$ is the velocity of the body in section $j$, $u_{s}$ is the solid flow rate, $C_{i j}$ is the fluid phase concentration of component $i$ in section $j, q_{i j}$ is the solid phase concentration of component $i$ in section $j$, and $q^{*}{ }_{i j}$ is the solid phase concentration at equilibrium between the solid and liquid phases in component $i$ and section $j$. In an SMB system, there may be more than one adsorption bed in each section, and the region will change with the node switching. Thus, by redefining adsorption bed $k, v^{*}{ }_{k}$ is the flow rate of the fluid in bed $k$. Therefore, a TMB and an SMB can be converted to each other as follows:

$$
\begin{gathered}
\frac{\partial C_{i j}}{\partial t}=D_{i} \frac{\partial^{2} C_{i j}}{\partial x^{2}}-v_{j}^{*} \frac{\partial C_{i j}}{\partial x}-\frac{1-\varepsilon}{\varepsilon} k_{i}\left(q_{i j}^{*}-q_{i j}\right), \\
\frac{\partial q_{i k}}{\partial t}=k_{i}\left(q_{i k}^{*}-q_{i k}\right) .
\end{gathered}
$$

\section{NN Model}

Over the last three decades, the NN technique has been widely employed for system identification. ${ }^{(13,14)}$ Through a simple training procedure, an NN is able to develop a complex and nonlinear mapping between the input and output pairs of training data automatically. Such a well-trained NN model can then be used for system identification. A well-known NN structure is the multilayer feed-forward network shown in Fig. 3. In our study, a four-layer NN with size 7-25-24-2 is mainly taken as the identification model. The network consists of one input layer with seven nodes, two hidden layers, one with 25 nodes and one with 24 nodes, and one output layer with two nodes. One output node identifies the concentration of material $A$ (caffeine) at the extract outlet and the other identifies the concentration of material $B$ (salicylic acid) at the raffinate outlet. In the NN model used, the sigmoid function is taken as the node's transfer function and the error back-propagation (BP) algorithm is taken as the learning rule for network training. ${ }^{(15)}$ 


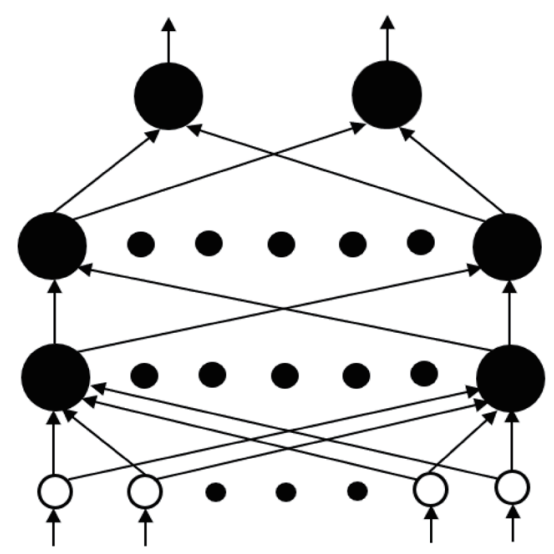

Fig. 3. Multilayer feed-forward NN.

\section{Experiments}

In this research, we perform experiments on the separation of two materials (caffeine and salicylic acid). The 2/2/2 SMB configuration shown in Fig. 4 is used to collect the relevant study data. In the SMB structure, there are three main sections, with each section having two subsections. The mixture is fed into the feed port and the desorbent is added from the desorbent inlet. The caffeine is separated at the extract outlet and the salicylic acid is separated at the raffinate outlet. The inlet and outlet are switched at each switching time.

In the separation process of the SMB, four parameters, namely, switching time $(T)$, desorbent flow rate $(D)$, extract flow rate $(E)$, and feed flow rate $(F)$, are recorded and collected. The percentage variation range of each parameter is listed in Table 1 and the values of each parameter used in the experiments are listed in Table 2.

In total, we collect 29160 data based on three switching times (171, 180, and 189) and various values of the other parameters. This data set is used for NN training. 3240 data based on switching times of 175 and 185 and various values of the other parameters are then collected and used for testing.

As mentioned above, a 7-25-24-2 NN is used for the SMB identification model. The inputs and outputs of the $\mathrm{NN}$ are listed as follows.

NN inputs:

Time (s), $T$ (s) (switching time), $C_{f a}\left(5 \mathrm{~kg} / \mathrm{m}^{3}\right)$ (caffeine concentration at feed port), $C_{f s}$ $\left(5 \mathrm{~kg} / \mathrm{m}^{3}\right)$ (salicylic acid concentration at feed port), $D\left(\mathrm{~m}^{3} / \mathrm{s}\right)$ (desorbent flow rate), $E\left(\mathrm{~m}^{3} / \mathrm{s}\right)$ (extract flow rate), and $F\left(\mathrm{~m}^{3} / \mathrm{s}\right)$ (feed flow rate).

NN outputs:

Separated caffeine concentration $C_{a}\left(\mathrm{~kg} / \mathrm{m}^{3}\right)$ and salicylic acid concentration $C_{S}\left(\mathrm{~kg} / \mathrm{m}^{3}\right)$.

To demonstrate the superiority of the NN in SMB identification, the training data are randomly reorganized into four groups with different training sequences. The mean squared 


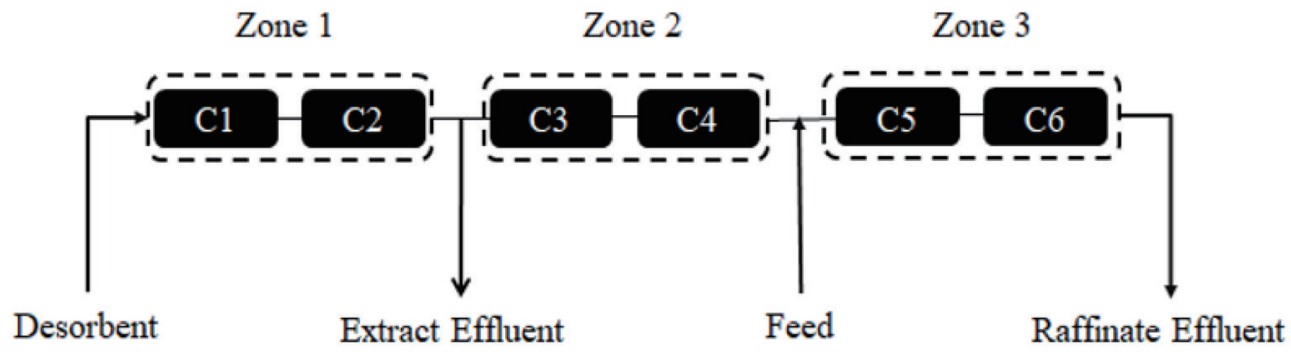

Fig. 4. 2/2/2 SMB configuration.

Table 1

Percentage variation range of each parameter.

\begin{tabular}{lcccc}
\hline Parameter & $T$ & $D$ & $E$ & $F$ \\
\hline Range & $\pm 5 \%$ & $\pm 5 \%$ & $\pm 5 \%$ & $\pm 20 \%$ \\
\hline
\end{tabular}

Table 2

Values of each parameter used in the experiment.

\begin{tabular}{lccccc}
\hline Variation & $-5 \%$ & $-3 \%$ & $0 \%$ & $3 \%$ & $5 \%$ \\
\hline$T(\mathrm{~s})$ & 171 & 175 & 180 & 185 & 189 \\
$D\left(\mathrm{~m}^{3} / \mathrm{s}\right)$ & $1.108 \times 10^{-7}$ & $1.132 \times 10^{-7}$ & $1.167 \times 10^{-7}$ & $1.202 \times 10^{-7}$ & $1.225 \times 10^{-7}$ \\
$E\left(\mathrm{~m}^{3} / \mathrm{s}\right)$ & $5.542 \times 10^{-8}$ & $5.658 \times 10^{-8}$ & $5.833 \times 10^{-8}$ & $6.008 \times 10^{-8}$ & $6.125 \times 10^{-8}$ \\
Variation & $-20 \%$ & $-15 \%$ & $-10 \%$ & $-5 \%$ & $0 \%$ \\
$F\left(\mathrm{~m}^{3} / \mathrm{s}\right)$ & $2 \times 10^{-8}$ & $2.15 \times 10^{-8}$ & $2.25 \times 10^{-8}$ & $2.375 \times 10^{-8}$ & $2.5 \times 10^{-8}$ \\
Variation & $5 \%$ & $10 \%$ & $15 \%$ & $20 \%$ & - \\
$F\left(\mathrm{~m}^{3} / \mathrm{s}\right)$ & $2.625 \times 10^{-8}$ & $2.75 \times 10^{-8}$ & $2.875 \times 10^{-8}$ & $3 \times 10^{-8}$ & - \\
\hline
\end{tabular}

Table 3

MSEs of training and test for four groups performed by NN.

\begin{tabular}{lcccc}
\hline Material & \multicolumn{2}{c}{ Caffeine } & \multicolumn{2}{c}{ Salicylic Acid } \\
\hline & Training MSE & Test MSE & Training MSE & Test MSE \\
\hline Group 1 & 0.001422 & 0.001499 & 0.000075 & 0.000092 \\
Group 2 & 0.001409 & 0.001265 & 0.000065 & 0.000159 \\
Group 3 & 0.001469 & 0.001729 & 0.000073 & 0.000063 \\
Group 4 & 0.001461 & 0.001587 & 0.000076 & 0.000054 \\
\hline Avg. & 0.001440 & 0.001520 & 0.000072 & 0.000092
\end{tabular}

error (MSE) is used to measure the NN performance. Table 3 presents the MSEs of training and test for Groups 1 to 4 performed by the NN.

Figures 5(a)-5(d) display the distributions of the estimated caffeine concentration obtained by the NN for the data in Groups 1 to 4, respectively. The distributions of the estimated salicylic acid concentration obtained by the NN for Groups 1 to 4 are shown in Figs. 6(a)-6(d), respectively.

Figure 7 shows the Group 1 test result of caffeine obtained by the well-trained NN, and the corresponding result of salicylic acid is shown in Fig. 8.

As seen on the graphs in Figs. 5-8, a well-trained NN can indeed accurately predict the concentration of substances to be separated by the SMB system. It also means that an NN can indeed be used for SMB system identification. 


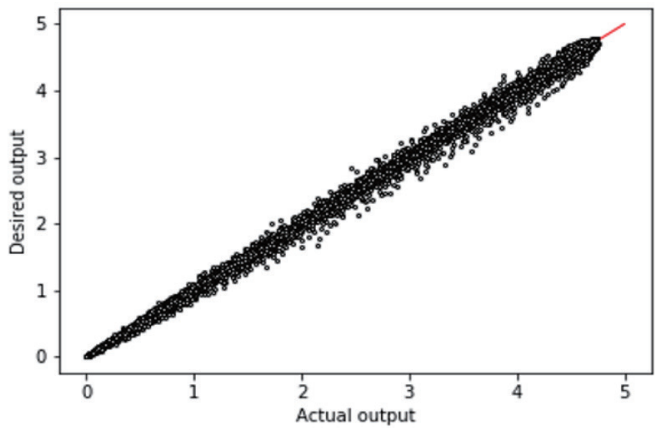

(a)

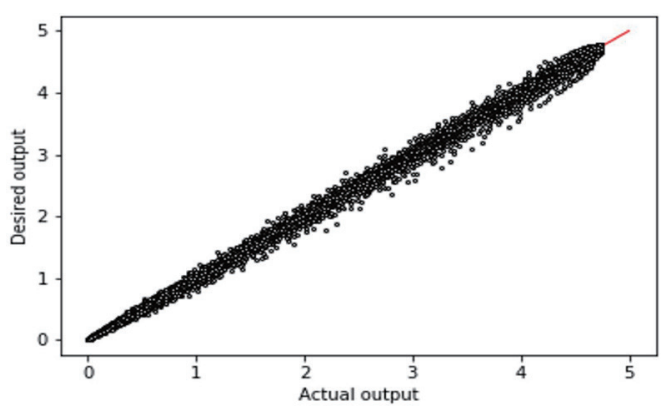

(c)

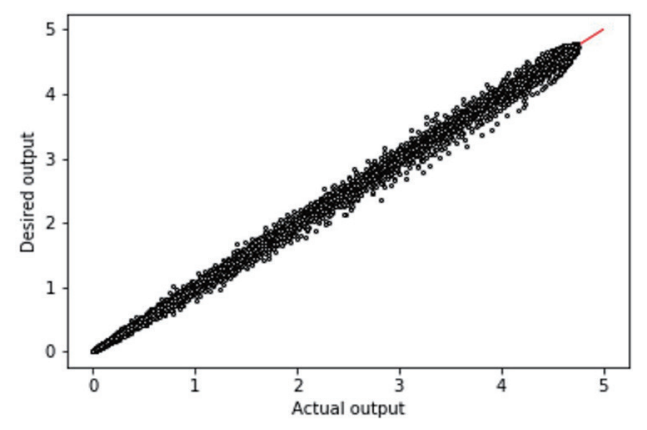

(b)

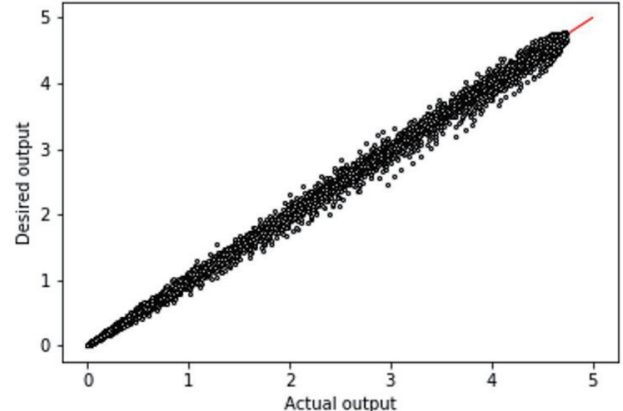

(d)

Fig. 5. (Color online) Distributions of estimated caffeine concentration obtained by NN for Groups 1 to 4.

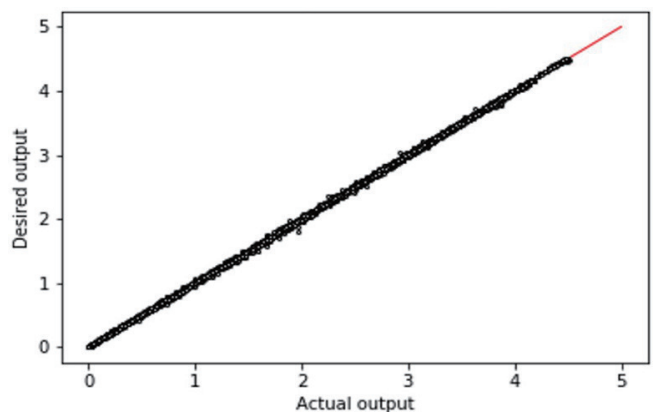

(a)

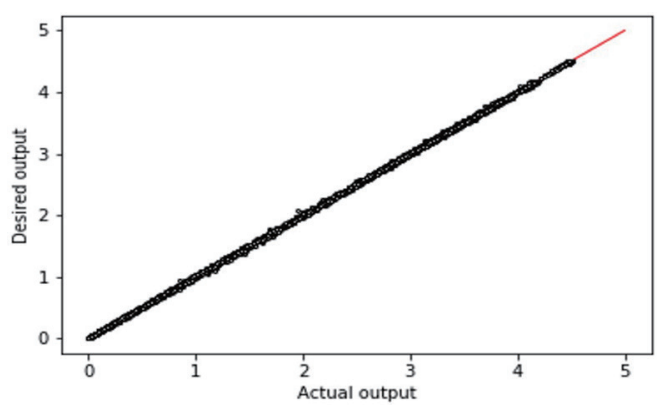

(c)

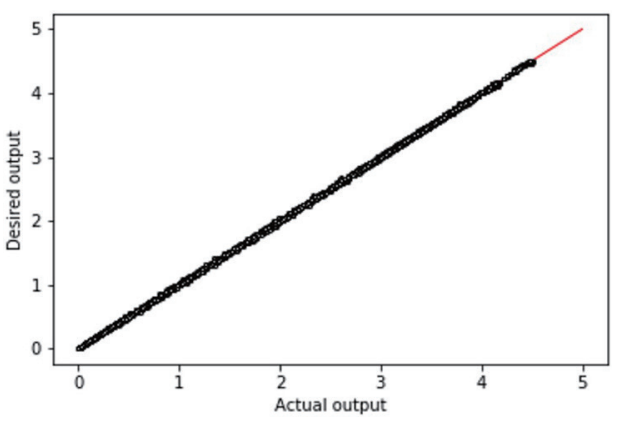

(b)

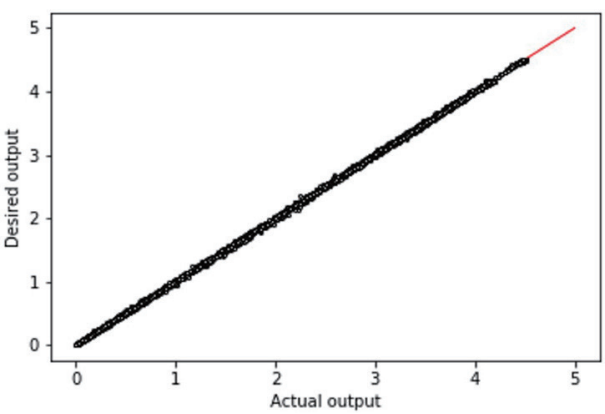

(d)

Fig. 6. (Color online) Distributions of estimated salicylic acid concentration obtained by NN for Groups 1 to 4 . 


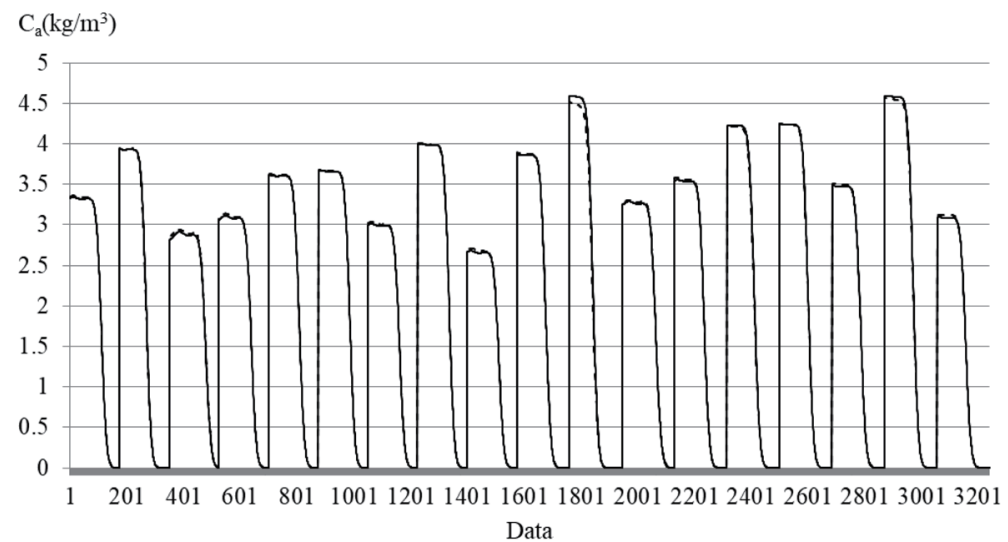

Fig. 7. Superposition diagram of NN test for caffeine concentration (Group 1). Solid line: desired output. Dashed line: actual output.

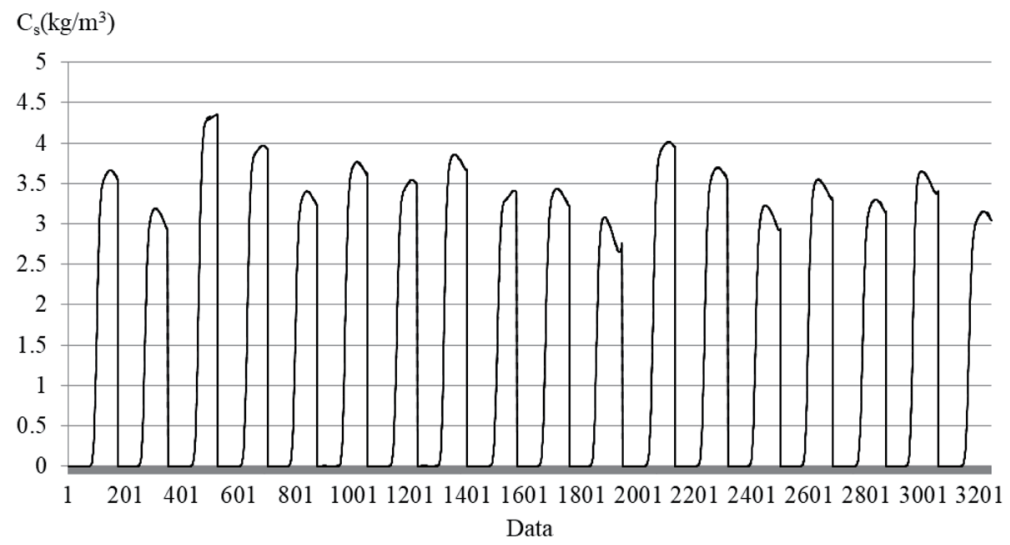

Fig. 8. Superposition diagram of NN test for salicylic acid concentration (Group 1). Solid line: desired output. Dashed line: actual output.

\section{Conclusions}

We present a means of identifying a SMB by the NN technique. To provide evidence that NN technology can be used for SMB system identification, we collected data sensed during the separation of two substances (caffeine and salicylic acid) by an SMB. These data represent the actual operation of the SMB separation process. The data collected on the basis of three switching times $(171,180$, and 189) were used to train the $\mathrm{NN}$, and the data collected on the basis of switching times 175 and 185 were then used to test the NN. Four randomly reorganized data groups were used to show the superiority of the NN in SMB identification. From the experimental results, the average test MSE of the NN for the caffeine concentration was $0.001520 \mathrm{~kg} / \mathrm{m}^{3}$ and that for the salicylic acid concentration was $0.000092 \mathrm{~kg} / \mathrm{m}^{3}$. This shows that the NN indeed could identify the complex behavior of the SMB system. The outputs generated by the $\mathrm{NN}$ were very close to the actual results of chromatographic separation by the 
SMB system. In other words, an NN is a very effective tool for SMB system identification, and a well-trained NN model could replace a real SMB system and be used for developing a precise control mechanism for an SMB without knowing its mathematical model. Thus, we conclude that it is possible to develop an intelligent controller with self-learning and adaptive abilities that can control an SMB to optimize the chromatographic separation process.

\section{Acknowledgments}

This research was supported by the Ministry of Science and Technology, Taiwan, R.O.C., under Contract No. MOST-107-2221-E-214-029.

\section{References}

1 A. Rajendran, G. Paredes, and M. Mazzotti: J. Chromatogr. A. 1216 (2009) 709. https://doi.org/10.1016/ j.chroma.2008.10.075

2 M. Schulte and J. Strube: J. Chromatogr. A. 906 (2001) 399. https://doi.org/10.1016/S0021-9673(00)00956-0

3 C. Y. Chin and N. L. Wang: Sep. Purif. Rev. 33 (2004) 77. https://doi.org/10.1081/SPM-200042081

4 A. E. Ribeiro, P. S. Gomes, L. S. Pais, and A. E. Rodrigue: Sep. Sci. Technol. 46 (2011) 1726. https://doi.org/10 $.1080 / 01496395.2011 .582070$

5 A. S. Andrade Neto, A. R. Secchi, M. B. Souza Jr, and A. G. Barreto Jr: J. Chromatogr. A. 1470 (2016) 42. https://doi.org/10.1016/j.chroma.2016.09.070

6 S. Mun and N. H. L. Wang: J. Chromatogr. A. 1488 (2017) 104. https://doi.org/10.1016/j.chroma.2016.12.052

7 Y. Yang, X. Chen, and N. Zhang: Adsorpt. Sci. Technol. 36 (2018) 1716. https://doi. org $/ 10.1177 / 0263617418804001$

8 M. A. F. Martins, A .C. Zanin, and D. Odloak: Chem. Eng. Res. Des. 92 (2014) 917. https://doi.org/10.1016/ j.cherd.2013.08.005

9 I. B. R. Nogueria, A. M. Ribeiro, M. A. F. Martins, A. E. Rodrigues, H. Koivisto, and J. M. Loureiro: J. Chromatogr. A. 1504 (2017) 112. https://doi.org/10.1016/j.chroma.2017.04.060

10 J. W. Lee and A. Seidel-Morgenstern: IFAC-Papers On Line. 51 (2018) 530. https://doi.org/10.1016/ j.ifacol.2018.09.370

11 M. T. Liang, R. C. Liang, L. R. Huang, K. Y. Liang, Y. L. Chieh, and J. Y. Liao: J. Taiwan Inst. Chem. Eng. 45 (2014) 1225. https://doi.org/10.1016/j.jtice.2013.10.013

12 M. T. Liang, R. C. Liang, L. R. Huang, P. H. Hsu, Y. H. Wu, and H. E. Yen: Am. J. Anal. Chem. 3 (2012) 931. https://doi.org/10.4236/ajac.2012.312A123

13 T. A. Tutunji: Appl. Soft Comput. 47 (2016) 251. https://doi.org/10.1016/j.asoc.2016.05.012

14 R. H. Abiyev: Int. J. Comput. Inf. Eng. 1 (2007) 2308. https://doi.org/10.5281/zenodo.1085665

15 C. Y. Shen, C. L. Hsu, R. C. Hwang, and J. S. Jeng: Sens. Actuators, B 122 (2007) 457. https://doi.org/10.1016/ j.snb.2006.06.017

\section{About the Authors}

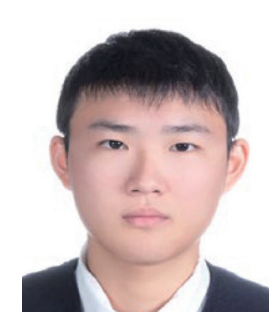

I-Chun Chen graduated from the Department of Electrical Engineering of I-Shou University. Currently, he is pursuing his Ph.D. degree in electrical engineering. His research interests are in artificial intelligence, fuzzy control, and signal processing. (qe660212@gmail.com) 


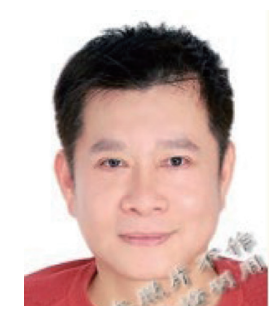

Huang-Chu Huang received his Ph.D. degree in electrical engineering from National Sun Yat-Sen University, Taiwan, in 2001. Currently, he is a professor of the Telecommunication Department, National Kaohsiung University of Science and Technology, Kaohsiung City, Taiwan. His research interests are in the areas of control, power signal prediction, and neural network applications. (h4530@nkust.edu.tw)

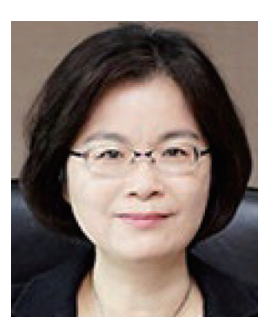

Chi-Yen Shen received her B.S. degree in electrical engineering from Cheng-Kung University, Taiwan, in 1987 and her Ph.D. degree in electrical engineering from National Sun Yat-Sen University, Taiwan, in 1991. She is currently a professor of electrical engineering at I-Shou University, Taiwan. Her research interests are in surface acoustic wave devices, sensors, and electronic ceramics. (cyshen@isu.edu.tw)

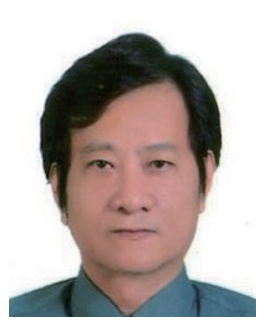

Rey-Chue Hwang received his Ph.D. degree in electrical engineering from Southern Methodist University, Dallas, TX, in 1993. Currently, he is a full professor of the Electrical Engineering Department, I-Shou University, Taiwan, R.O.C. Dr. Hwang has published more than 300 papers in various journals and conferences in the areas of artificial intelligence systems, signal processing, and fuzzy control. He is now a fellow of IET and a senior member of IEEE. He chartered the IEEE CIS Chapter, Tainan Section, and served as the co-chair and chair from 2004 to 2009. 